\title{
Acute One-Stop Cardiovascular Magnetic Resonance Imaging for Differential Diagnosis in Patients with Acute Coronary Syndrome and Unobstructed Coronary Arteries
}

\author{
Guang Chu $^{\mathrm{a}}$ Guobing Zhang ${ }^{\mathrm{a}}$ Ming Zhu $^{\mathrm{b}}$ Zhi Zhang ${ }^{\mathrm{a}}$ Ying Wu $^{\mathrm{a}}$ Hao Zhang ${ }^{\mathrm{b}}$ \\ Departments of a Cardiology and ${ }^{b}$ Radiology, Shanghai Jiaotong University, First People's Hospital, Shanghai, \\ People's Republic of China
}

\section{Key Words}

Acute coronary syndrome - Chest pain .

Magnetic resonance imaging $\cdot$ Coronary artery disease

\begin{abstract}
Objective: We aimed to evaluate the contributions of acute one-stop cardiovascular magnetic resonance (CMR) imaging to the differential diagnosis of acute coronary syndrome (ACS) and unobstructed coronary arteries. Subjects and Methods: In this study, 32 consecutive patients who presented with ACS and unobstructed coronary arteries on angiography were enrolled between January 2010 and December 2012. Acute one-stop CMR, including cine, angiography, black-blood, first-pass perfusion and late gadolinium enhancement (LGE) imaging, was performed with a pre-specified algorithm which was decided on by the doctors for all patients. The intimal flap in the aorta and the filling defect in the pulmonary artery were detected on MR angiography imaging. Left ventricular wall motion and ventricular thickness were analyzed in cine-mode sequences. The LGE images were reviewed for the presence, anatomical distribution and extent of contrast enhancement. Results: The acute onestop CMR study was completed in all the 32 patients without
\end{abstract}

adverse events. The overall time duration was between 15 and $60 \mathrm{~min}$. Of the 32 patients, a CMR diagnosis was made in 30 (93.8\%). Aortic dissection was detected in 3 patients, pulmonary embolism in 2, hypertrophic cardiomyopathy in 2 , acute myocardial infarction in 5 , acute myocarditis in 16 and stress cardiomyopathy in 2 . No confirmed diagnosis was established in the remaining 2 patients with normal CMR. Conclusion: Acute one-stop CMR allowed for the identification of an aetiology in most of the patients in this study. It may prove to be of immense help in establishing a differential diagnosis in patients presenting with acute chest pain, elevated troponin I and normal coronary arteries.

(c) 2015 S. Karger AG, Basel

\section{Introduction}

Patients who arrive at hospital with prolonged chest pain, elevated levels of the biomarkers of myocardial damage and abnormal electrocardiography (ECG) are admitted with an initial diagnosis of acute coronary syn-

Guang Chu and Hao Zhang contributed equally to this work.

\begin{tabular}{ll}
\hline KARGER 125/s & $\begin{array}{l}\text { ○ 2015 S. Karger AG, Basel } \\
1011-7571 / 15 / 0244-0325 \$ 39.50 / 0 \quad \text { Karger }\end{array}$ \\
E-Mail karger@karger.com & $\begin{array}{l}\text { This is an Open Access article licensed under the terms of the } \\
\text { Creative Commons Attribution-NonCommercial 3.0 Un- } \\
\text { ported license (CC BY-NC) (www.karger.com/OA-license), } \\
\text { applicable to the online version of the article only. Distribu- } \\
\text { tion permitted for non-commercial purposes only. }\end{array}$
\end{tabular}

Guobing Zhang, $\mathrm{MD}, \mathrm{PhD}$

Department of Cardiology, Shanghai Jiaotong University First People's Hospital

85 Wu Jin Road, Shanghai 200080 (People's Republic of China)

E Mail tq20052005@126.com 
drome (ACS) [1]. It is necessary for high-risk patients to undergo primary percutaneous coronary angioplasty. Approximately $10 \%$ of these patients do not have significant coronary artery stenosis $[1,2]$; this represents a serious diagnostic challenge. Although the situation is sometimes one of acute myocardial infarction (AMI) without the angiographic features of coronary lesions, other nonischaemic cardiovascular diseases such as pulmonary embolism (PE), aortic dissection (AD) or acute myocarditis can present a clinical picture similar to ACS $[3,4]$. Establishing a correct diagnosis has significant prognostic and therapeutic implications for such patients.

Cardiovascular magnetic resonance (CMR) is a noninvasive, comprehensive imaging technique that allows for the simultaneous assessment of cardiovascular anatomy, tissue characterization and functional analysis [2]. Gadolinium can be utilized for high-resolution pulmonary and aortic magnetic resonance angiography (MRA) to exclude $\mathrm{PE}$ or $\mathrm{AD}[5,6]$. Cardiac dimensions, hypertrophy patterns and motion abnormalities can be easily visualized in cine-mode sequences [7, 8]. In addition, inflammation, myocardial hypoperfusion and infarct-related necrosis are distinctly detectable using $\mathrm{T}_{2}$-oedema or late gadolinium enhancement (LGE) imaging techniques [9-11]. Finally, CMR is playing an increasingly important role in the initial diagnosis and management of various cardiovascular diseases [12].

In this study, we sought to implement acute one-stop $\mathrm{CMR}$ as a non-invasive imaging method for differential diagnosis in patients presenting with ACS and unobstructed coronary arteries.

\section{Subjects and Methods}

Of the 1,215 patients who were admitted to the Department of Cardiology with an initial diagnosis of ACS and who underwent primary coronary angiography (CAG) between January 2010 and December 2012, 118 presented with unobstructed coronary angiograms. Of these 118 eligible patients, 75 were admitted outside the CMR operational hours, 8 were contraindicative of CMR and 3 had a history of myocardial infarction or hypertrophic cardiomyopathy. Hence, 32 patients (age range $24-78$ years) were enrolled in the study. They presented with an acute onset of chest pain ( $>30 \mathrm{~min}$ ), elevated levels of cardiac troponin I $(>0.1 \mathrm{ng} / \mathrm{ml})$, ECG changes indicative of new ischaemia (new ST-T changes, the development of pathological Q waves or new left bundle branch block) and unobstructed coronary arteries (stenosis $<50 \%$ of the diameter of the vessel) on angiography. Patients were excluded when they were admitted outside the CMR working hours (between 6 p.m. and 8 a.m. or on a Sunday) and when they had acute heart failure, critical arrhythmia, an unstable haemodynamic condition, a history of myocardial infarction or hypertrophic cardio- myopathy, claustrophobia and implanted pacemakers or other metal devices. All the enrolled patients underwent serial ECG, serial measurement of myocardial damage marker levels, chest Xray, CAG and one-stop CMR. Primary CAG was performed no later than $2 \mathrm{~h}$ after admission. Acute CMR was conducted 2-4 h immediately after negative cardiac catheterization. The study was approved by the Institutional Ethics Committee and Review Board of the hospital.

\section{Acute One-Stop CMR Study}

All one-stop CMR procedures were performed using a Signa EXCITE 1.5-tesla clinical imaging system (GE Medical Systems, Milwaukee, Wis., USA) that provides the possibility of monitoring blood pressure, heart rate and blood oxygen saturation simultaneously. The pre-specified algorithm for stepwise evaluation was decided by the authors. The algorithm could be followed step-by-step or interrupted at each point to minimize the examination time for the patient.

First, due to the severe life-threatening risk of $\mathrm{AD}$ and $\mathrm{PE}$, these were ruled out before the cardiac MR was conducted. Which examination was performed first depended on the symptoms of the patient. After scout and reference scans, 6 steady-state free-precession (SSFP) cine images with parallel imaging (slice thickness: 8 mm and sense factor: 2 ) were acquired through the thoracic aorta with subsequent MRA if $\mathrm{AD}$ was suspected. The non-breath-hold SSFP sequence scans, contrast-enhanced 3-dimensional (3D)-MR pulmonary angiography (half-scan data acquisition; rec voxel: $0.7 / 0.7 / 2 \mathrm{~mm}$ and sense factor: 2 ) and $3 \mathrm{D}$ gradient-echo (GRE) imaging were performed using double-dose gadolinium-DPTA if PE was suspected.

The one-stop CMR study ended when the diagnosis of $\mathrm{AD}$ or PE was confirmed; otherwise, cardiac MR was performed. After scout views to determine cardiac axis locations, the typical protocol involved 4 stages.

\section{Cine Mode}

The SSFP sequences were acquired in 3 cardiac conventional planes [left ventricular (LV) long-axis, short-axis and 4-chamber view] with these typical settings: breath-hold acquisition/each slice, a slice thickness of 7-10 mm with no gap, the shortest TR/TE typically $3.7 / 1.6 \mathrm{~ms}$, a flip angle of $45^{\circ}$, a field of view (FOV) of $320-380 \mathrm{~mm}$ and a matrix of $224 \times 192$. In the short-axis view, 10-12 slices were acquired with complete coverage of the left ventricle from base to apex.

\section{Double/Triple Inversion Recovery}

Double/triple inversion recovery (IR) preparation was done in the dual-contrast fast-spin echo sequence. The double IR preparation consisted of a non-selective adiabatic inversion pulse followed immediately by a selective inversion pulse to suppress the blood signal. This is similar to $\mathrm{T}_{1}$-weighted imaging. The triple IR preparation consisted of the double IR pulse and an extra radiofrequency pulse to suppress the fat signal. This is similar to $\mathrm{T}_{2}$-weighted imaging. The dual contrast was performed through a split-echo train. The following imaging parameters were applied: a TE1 of 42 ms, a TE2 of $102 \mathrm{~ms}$, echo spacing of $4.2 \mathrm{~ms}$, a TI of $575 \mathrm{~ms}$, a TR of $2 \times \mathrm{R}-\mathrm{R}$ intervals, an FOV of $320-400 \mathrm{~mm}$, a matrix of $256 \times 256$ and a slice thickness of $8-10 \mathrm{~mm}$ without a gap. Eight contiguous slices in a short-axis view were placed to cover the LV basal to apical planes by breath holding during each section. 
First-Pass Perfusion Imaging

Short-axis, first-pass perfusion CMR was performed using a $\mathrm{T}_{1}$-weighted fast GRE sequence with saturation-recovery magnetization preparation (FGRET) with these typical settings: 8 contiguous slices in the short-axis view placed to cover the LV basal to apical planes and recorded continuously for each cardiac cycle, a slice thickness of 8-10 mm without an intersection gap, the shortest TR/TE typically $7.6 / 2.4 \mathrm{~ms}$, a matrix of $128 \times 128$, an FOV of 270-360 $\mathrm{mm}$. The total duration was approximately $1 \mathrm{~min}$ with partial breath holding during the initial myocardial enhancement. The acquisition was synchronized with the intravenous injection of $0.1 \mathrm{mmol} / \mathrm{kg}$ of gadolinium-DPTA at a rate of $3.5-4 \mathrm{ml} / \mathrm{s}$, followed by a flush infusion at the same rate.

\section{LGE Imaging}

After perfusion imaging, an additional dose of $0.1 \mathrm{mmol} / \mathrm{kg}$ of gadolinium-DPTA was administered at a rate of $0.5 \mathrm{ml} / \mathrm{s}$. With a 12-min delay, LGE MRI images were acquired with breath-holding for each stack, utilizing an IR-prepared, segmented GRE sequence. These settings were typically: a stack of 8 contiguous slices in the short-axis view with the orientations identical to perfusion imaging, a slice thickness of $8-10 \mathrm{~mm}$ without an intersection gap, a $\mathrm{TR} / \mathrm{TE} /$ inversion time of 4.0/1.8/200-360 ms, an FOV of 320-360 $\mathrm{mm}$, a matrix of $192 \times 160$ and a flip angle of $20^{\circ}$.

The cardiac MR images were assessed by 2 experienced radiologists (H.Z. and M.Z.). Ventricular volumes, LV ejection fraction (LVEF) and ventricular thickness were calculated. LV wall motion was measured according to the classification of the American Heart Association [13]. $\mathrm{T}_{2}$-weighted images were used to detect potential areas of high signal intensity consistent with myocardial oedema, which were measured by visual estimation without any quantification method. The presence of microvascular obstruction was visually ascertained on first-pass perfusion images. Finally, the LGE images were also visually reviewed for the presence, anatomical distribution and extent of subendocardial, subepicardial, mid-wall and transmural contrast enhancement. The final diagnosis was based on the delayed contrast-enhancement images. A diagnosis of AMI was made from images that showed areas of subendocardial and transmural contrast enhancement, while diagnoses of other myocardial diseases were made with subepicardial and intramyocardial contrast enhancement.

\section{Results}

The baseline characteristics of the 32 patients who ultimately underwent acute CMR are presented in table 1. All of the patients completed the CMR imaging without adverse events. The mean duration time of one-stop CMR was $42.5 \pm 18.2 \mathrm{~min}$ (range 15-60 $\mathrm{min}$ ) and the findings are presented in table 2 . Of the 32 patients, a CMR diagnosis was made in 30 (93.8\%). The details of the differential diagnosis are summarized in table 3 . Three patients were diagnosed with $\mathrm{AD}$. The intimal flap that leads to a widened false lumen of the thoracic aorta and the broad entry as a communication between the two lumens was
Table 1. Baseline characteristics of the study population $(n=32)$

Age, years

$47.5 \pm 18.2$

Men

$18(56.3)$

Cardiovascular risk factors

Smoking

$20(62.5)$

Diabetes

$13(40.6)$

Hyperlipidaemia

$10(31.3)$

Obesity

$16(50.0)$

Hypertension

$15(46.9)$

Family history

$14(43.8)$

Serum parameters

Cardiac troponin I admission, $\mathrm{ng} / \mathrm{ml} \quad 8.3 \pm 6.2$

C-reactive protein, $\mathrm{mg} / \mathrm{l}$

$42.5 \pm 21.5$

D-dimer, mg/l

$1.8 \pm 5.3$

ST-segment elevation

$13(40.6)$

ST-segment depression

$7(21.9)$

T-wave abnormalities

$5(15.6)$

Pathological Q waves

$3(9.4)$

$1(3.1)$

New left bundle branch block

$3(9.4)$

Risk stratification

GRACE score

$135.3 \pm 38.6$

Values are $\mathrm{n}(\%)$ or mean \pm SD.

Table 2. Findings on cardiac MR $(n=27)$

$\begin{array}{lc}\text { Cine mode } & \\ \quad \text { LVEF } & 52.4 \pm 9.2 \\ \quad \text { Septal or apical segment hypertrophy } & 2(7.4) \\ \quad \text { Wall motion abnormalities } & 17(63.0) \\ \mathrm{T}_{2} \text {-weighted spin-echo sequences } & \\ \quad \text { Oedema } & 14(51.9) \\ \text { First-pass perfusion imaging } & \\ \quad \text { Microvascular obstruction } & 1(3.7) \\ \text { Presence of LGE } & \\ \quad \text { Subendocardial } & 4(14.8) \\ \quad \text { Transmural } & 2(7.4) \\ \quad \text { Subepicardial } & 12(44.4) \\ \quad \text { Intramyocardial } & 5(18.5) \\ \text { Normal cardiac MR } & 2(7.4)\end{array}$

Values are $\mathrm{n}(\%)$ or mean \pm SD.

detected on ECG-triggered cine SSFP images taken in anatomical transversal orientation during the diastolic and systolic periods (fig. 1a). Normal flow in the narrow true lumen and extremely slow flow within the false lumen were observed on dynamic contrast-enhanced 3D-MRA of the aorta taken in coronal orientation. The filling defect or vascular truncation signs in the left and right pul- 

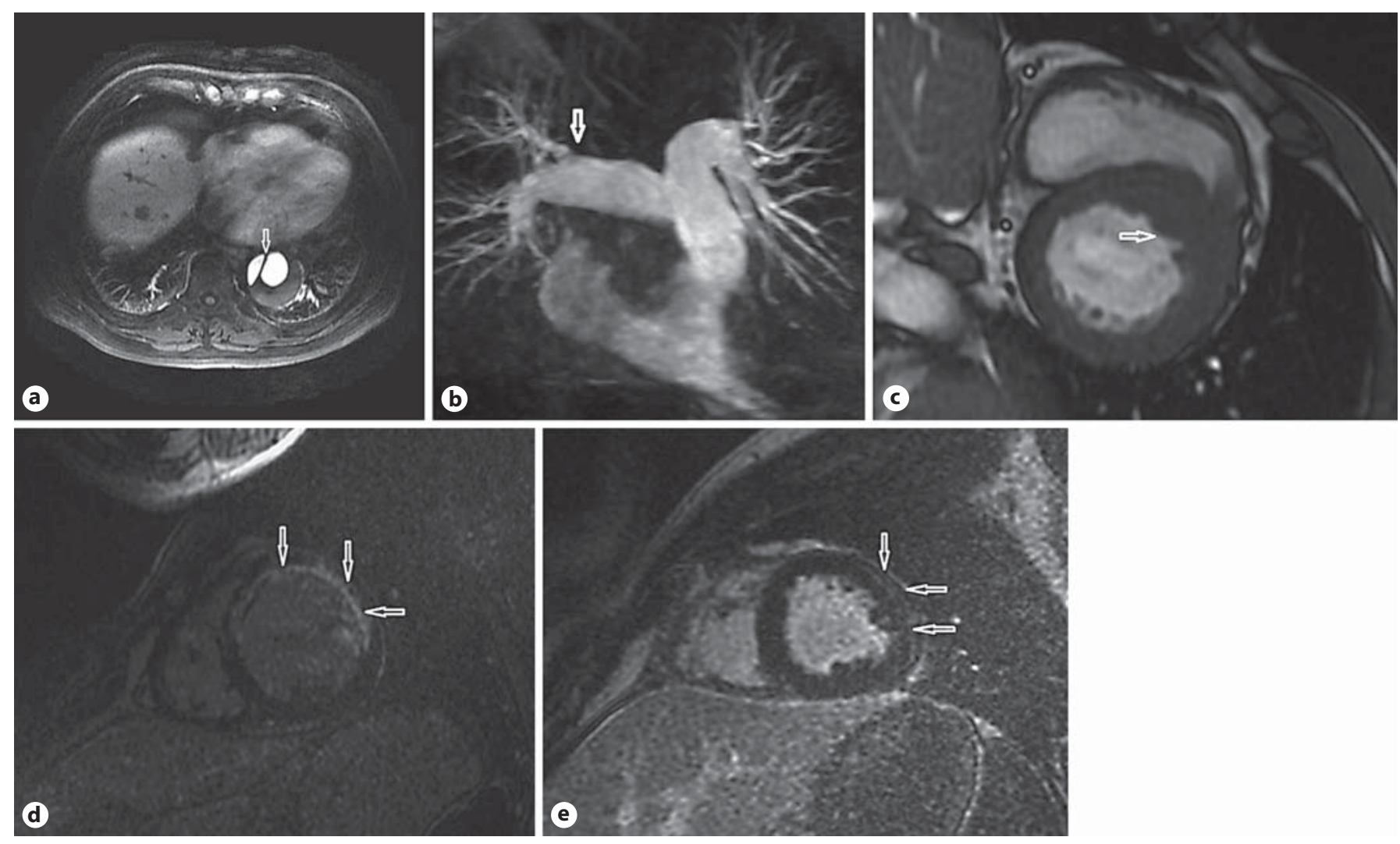

Fig. 1. CMR images of the patients suspected of having ACS. a Contrast-enhanced $\mathrm{T}_{1}$-weighted images taken in an anatomical transversal orientation showing an intimal flap (arrow) in the thoracic aorta. b 3D-MR pulmonary angiography showing filling-defect signs (arrow) in the right upper lobe pulmonary arterial branch. c SSFP images in the short-axis view showing asymmetric increased thickness (arrow) at the base of the interventricular septum. d IR images in the short-axis view of the left ventricle showing transmural delayed contrast enhancement (arrows) in the extensive anterior wall. e IR images in the short-axis view of the left ventricle showing subepicardial delayed contrast enhancement (arrows) in the lateral wall.
Table 3. Final diagnosis from the CMR study $(n=32)$

\begin{tabular}{lc}
\hline $\mathrm{AD}$ & $3(9.3)$ \\
$\mathrm{PE}$ & $2(6.3)$ \\
Hypertrophic cardiomyopathy & $2(6.3)$ \\
AMI & $5(15.5)$ \\
Acute myocarditis & $16(50.0)$ \\
Stress cardiomyopathy & $2(6.3)$ \\
No diagnosis & $2(6.3)$ \\
\hline
\end{tabular}

Values are $\mathrm{n}(\%)$.

monary arterial branch were detected on SSFP imaging, gadolinium chelate-based contrast-enhanced breathhold MR pulmonary angiography or recirculation-phase breath-hold 3D GRE imaging in 2 patients who had a diagnosis of PE (fig. 1b).
Two patients were diagnosed with hypertrophic cardiomyopathy, presenting as asymmetric increased thickness $(15-21 \mathrm{~mm})$ at the base of the interventricular septum or apical segment, and a significant reduction in the LV cavity on the SSFP images (fig. 1c). The punctate or patchy LGE was seen in the hypertrophic hypokinetic segments, which helped to distinguish it from AMI.

A hyperintense signal compatible with oedema was detected on $\mathrm{T}_{2}$-weighted images in 14 patients. It was located in the septal or anterior segments in 4 patients and in the lateral and lower segments in the remaining 10 . Areas of subendocardial contrast enhancement were detected on the delayed contrast-enhancement images in 3 patients, and areas of transmural contrast enhancement were detected in 2 patients, one with an anterior location and the other with an inferior location (fig. 1d). These 5 patients who presented subendocardial or transmural 
contrast enhancement were diagnosed with AMI; 1 of them displayed typical microvascular obstruction on the first-pass perfusion images, and 2 displayed a hyperintense signal compatible with myocardial oedema on the $\mathrm{T}_{2}$-weighted images.

Areas of subepicardial or intramyocardial contrast enhancement were mainly located in the LV lateral segments in 16 patients (fig. 1e). The contrast-enhancement pattern was multifocal and patchy in 12 patients, and 11 patients presented a hyperintense signal on $\mathrm{T}_{2}$-weighted images. These 16 patients were diagnosed as having acute myocarditis. Another CMR checkup 1 month later showed that the myocardial contrast signal had basically returned to normal in 6 patients.

There was no positive finding when using the contrast enhancement on the IR images in 4 patients. Two were post-menopausal women who had an exaggerated sympathetic stimulation with an antecedent stress event. They displayed severe contractile abnormalities in the apical segments with a low LVEF (30-35\%) on the cinemode images and an absence of contrast enhancement, ruling out AMI or acute myocarditis. One of them had myocardial oedema on $\mathrm{T}_{2}$-weighted imaging. Thus, the diagnosis of stress cardiomyopathy was clear. CMR reexamination showed that their LV dysfunction was completely recovered and the LVEF was $56-60 \%$ at 14 days, which further confirmed the initial diagnosis made on CMR. No confirmed diagnosis was established in the remaining 2 patients on normal CMR during the early stage of hospitalization.

\section{Discussion}

There is a broad consensus that CMR imaging, especially with LGE has the ability to differentiate conditions that closely mimic ACS [12]. In this study, acute one-stop CMR enabled the aetiological origin of the clinical picture to be identified in most (93.8\%) of the patients versus $90-95 \%$ in patients with ACS and normal coronary arteries as reported in 2 previous studies by Laraudogoitia Zaldumbide et al. [2] and Leurent et al. [14], respectively. However, it must be noted that there are some differences in the research scheme between our study and others.

Compared with some other studies [2, 14-16] in which the one-stop CMR was conducted several days after the primary CAG, in our study it was performed just 2-4 h afterwards. Establishing an accurate diagnosis as quickly as possible has paramount importance for both the prognosis and therapeutic outcome in patients suffering from a potentially life-threatening condition. Treatments for different diseases differ immensely. Antithrombotic treatment was withdrawn after acute CMR in $78.1 \%$ of the patients in our series who did not have AMI or PE, $6.2 \%$ of the PE patients received thrombolytic therapy and $9.7 \%$ of the $\mathrm{AD}$ patients were transferred to the Department of Vascular Surgery for an operation. The other explanation could be that the extension of the interval between the onset of symptoms and CMR imaging meant that several transient abnormalities detectable on CMR (e.g. dyskinetic wall motion, pericardial effusion and myocardial oedema) might have resolved. This would reduce the diagnostic value of CMR. Definite diagnoses were made on the basis of acute CMR imaging in nearly $95 \%$ of our patients whereas Assomull et al. [15] reported that the overall diagnostic contribution of CMR in their patients was only $65 \%$, probably due to the median delay of 14.5 days between the onset of chest pain and CMR.

Due to the high mortality and morbidity associated with $\mathrm{AMI}, \mathrm{PE}$ and $\mathrm{AD}$, these 3 diseases should be ruled out first in patients presenting with acute chest pain and elevated troponin I. Therefore, after negative CAG, thoracic aorta MR and pulmonary MR were conducted prior to cardiac MR in our series. This procedure was rarely mentioned in previous studies $[2,14]$, although echocardiography was conventionally performed before CMR. Echocardiography is able to detect thrombi in the pulmonary and intimal flap in the thoracic aorta. Sensitivity and specificity are very low in detecting $\mathrm{AD}$ and $\mathrm{PE}$, but contrast-enhanced 3D-MRA of the aorta, pulmonary MRA and 3D GRE imaging are much more sensitive and specific $[5,6,17,18]$. Three cases of $\mathrm{AD}$ and 2 cases of $\mathrm{PE}$ were diagnosed clearly by the use of the above MR technique in this study. Furthermore, it should be noted that the experienced cardiologist decided on the examination procedures according to the clinical manifestations. For instance, pulmonary MR was performed primarily on the patients with hypoxaemia and elevated levels of D-dimer. This minimized the testing time and made early diagnosis possible for our patients.

In contrast to this study, Steen et al. [19] performed acute CMR before cardiac catheterization in the chest pain unit in 29 patients with elevated troponin I and a low-to-intermediate probability of ACS; they detected PE, AMI, myocarditis and cardiomyopathy step by step, with $37.9 \%(11 / 29)$ of their patients being given a diagnosis of AMI. The point is that approximately $30 \%$ of the patients with ACS did not have typical clinical manifesta- 
tions, conclusive ECG changes or cardiovascular risk factors [20]. Some patients do not have to undergo further invasive procedures after acute CMR, but others may miss the optimal opportunity to undergo primary percutaneous coronary intervention. However, the patients in our study had undergone CAG before the CMR examination. Most of the patients with AMI were ruled out, and the remaining patients had normal coronary flow, which reduced the risk of sudden cardiovascular events during the CMR study. It was indubitably safer and better tolerated. In addition, in view of non-coronary cardiac disease presenting with acute chest pain and the differential diagnosis of the underlying pathology, MRI has unique diagnostic features [21]. Therefore, in our series, acute CMR was conducted after CAG in patients suspected of having ACS.

In this study, no significant abnormality was seen on the CMR images of 2 patients with a modest increase in troponin $\mathrm{I}(1.2-2.4 \mathrm{ng} / \mathrm{ml})$ above the normal range, which is a sign of minimal myocardial necrosis. One of them had a final diagnosis of AMI, demonstrated by the typically dynamic changes on ECG and the biomarkers of myocardial damage in 1 week. The explanation may be that the absence of contrast enhancement on the CMR was due to the fact that the technique is limited by inadequate spatial resolution when detecting very small necrotic areas [22]. Three days after the onset of chest pain, the symptoms were completely alleviated and a moderate amount of pericardial effusion was detected on echocardiography at the same time in the other patient, who was suspected of having acute perimyocarditis. This could have been due to the above-mentioned reason, or it may have been that there was no wall motion abnormality or pericardial effusion at an early stage, which is nicely depicted in the black-blood-prepared $\mathrm{T}_{2}$-weighted images as well as in the SSFP cine loops [23].

There are some limitations to our study. First, since MRI services in our hospital are not round-the-clock, we only enrolled 32 patients with ACS and unobstructed coronary arteries. So the sample size in this single center was relatively small. Second, no long-term follow-up was implemented in any patients, and only a few patients underwent repeated CMR (this can yield prognostic information). Third, the criteria for deciding who would undergo the acute CMR study were not standardized, and the decision was left to the discretion of the cardiologist.

\section{Conclusion}

Acute one-stop CMR safely allowed for the identification of an aetiology in more than $90 \%$ of the patients in this study. It may prove to be of immense help in establishing a differential diagnosis in patients presenting with acute chest pain, elevated troponin I and normal coronary arteries.

\section{References}

1 Arai AE: False positive or true positive troponin in patients presenting with chest pain but 'normal' coronary arteries: lessons from cardiac MRI. Eur Heart J 2007;28:1175-1177.

-2 Laraudogoitia Zaldumbide E, Perez-David E, Larena JA, et al: The value of cardiac magnetic resonance in patients with acute coronary syndrome and normal coronary arteries. Rev Esp Cardiol 2009;62:976-983.

$>3$ Larson DM, Menssen KM, Sharkey SW, et al: 'False-positive' cardiac catheterization laboratory activation among patients with suspected ST segment elevation myocardial infarction. JAMA 2007;298:2754-2760.

$\checkmark 4$ Mahajan N, Mehta Y, Rose M, et al: Elevated troponin level is not synonymous with myocardial infarction. Int J Cardiol 2006;111:442449.
5 Kalb B, Sharma P, Tigges S, et al: MR imaging of pulmonary embolism: diagnostic accuracy of contrast-enhanced 3D MR pulmonary angiography, contrast-enhanced low-flip angle 3D GRE, and non-enhanced free-induction FISP sequences. Radiology 2012;63:271-278.

6 Krishnam MS, Tomasian A, Malik S, et al: Image quality and diagnostic accuracy of unenhanced SSFP MR angiography compared with conventional contrast-enhanced MR angiography for the assessment of thoracic aortic diseases. Eur Radiol 2010;20:13111320.

7 Proctor RD, Shambrook JS, McParland P, et al: Imaging hypertrophic heart diseases with cardiovascular MR. Clin Radiol 2011;66:176186.

$\checkmark 8$ Leurent G, Larralde A, Boulmier D, et al: Cardiac MRI studies of transient left ventricular apical ballooning syndrome (Takotsubo cardiomyopathy): a systematic review. Int J Cardiol 2009;135:146-149.
-9 Friedrich MG, Sechtem U, Schulz-Menger J, et al: Cardiovascular magnetic resonance in myocarditis: a JACC white paper. JACC 2009; 53:1475-1487.

10 Yalcinkaya E, Bugan B, Celik M, et al: Cardiomyopathies: the value of cardiac magnetic resonance imaging. Med Princ Pract 2014;23: 191.

11 Friedrich MG: Tissue characterization of acute myocardial infarction and myocarditis by cardiac magnetic resonance. JACC Cardiovasc Imaging 2008;1:652-662.

12 Bruder O, Schneider S, Nothnagel D, et al: Euro CMR (European Cardiovascular Magnetic Resonance) Registry: results of the German pilot phase. J Am Coll Cardiol 2009;54: 1457-1466. 
13 Cerqueira MD, Weissman NJ, Dilsizian V, et al: Standardized myocardial segmentation and nomenclature for tomographic imaging of the heart: a statement for healthcare professionals from the Cardiac Imaging Committee of the Council on Clinical Cardiology of the American Heart Association. Circulation 2002;105:539-542.

14 Leurent G, Langella B, Fougerou C, et al: Diagnostic contributions of cardiac magnetic resonance imaging in patients presenting with elevated troponin, acute chest pain syndrome and unobstructed coronary arteries. Arch Cardiovasc Dis 2011;104:161-170.

15 Assomull RG, Lyne JC, Keenan N, et al: The role of cardiovascular magnetic resonance in patients presenting with chest pain, raised troponin and unobstructed coronary arteries. Eur Heart J 2007;28:1242-1251.
16 Chopard R, Jehl J, Dutheil J, et al: Evolution of acute coronary syndrome with normal coronary arteries and normal cardiac magnetic resonance imaging. Arch Cardiovasc Dis 2011;104:509-517.

17 Ohno Y, Koyama H, Matsumoto K, et al: Dynamic MR perfusion imaging: capability for quantitative assessment of disease extent and prediction of outcome for patients with acute pulmonary thromboembolism. J Magn Reson Imaging 2010;31:1081-1090.

18 Nienaber CA: The role of imaging in acute aortic syndromes. Eur Heart J Cardiovasc Imaging 2013;14:15-23.

19 Steen H, Madadi-Schroeder M, Lehrke S, et al: Staged cardiovascular magnetic resonance for differential diagnosis of troponin $\mathrm{T}$ positive patients with low likelihood for acute coronary syndrome. J Cardiovasc Magn Reson 2010;12:51.
20 Bassand JP, Hamm CW, Ardissino D, et al: Guidelines for the diagnosis and treatment of non-ST-segment elevation acute coronary syndromes. Eur Heart J 2007;28:15981660.

21 Hunold P, Bischoff P, Barkhausen J, et al: Acute chest pain: the role of MR imaging and MR angiography. Eur J Radiol 2012;81:36803690.

22 Vohringer M, Mahrholdt H, Yilmaz A, et al: Significance of late gadolinium enhancement in cardiovascular magnetic resonance imaging (CMR). Herz 2007;32:129-137.

23 Sa MI, Kiesewetter CH, Jagathesan R, et al: Images in cardiovascular medicine. Acute pericarditis assessed with magnetic resonance imaging: a new approach. Circulation 2009; 119:e183-e186. 